\title{
Parosteal osteosarcoma dedifferentiating into telangiectatic osteosarcoma: importance of lytic changes and fluid cavities at imaging
}

\author{
Michael J. Klein
}

Published online: 14 October 2009

(C) ISS 2009

Dear Sir:

The scientific article entitled "Parosteal osteosarcoma dedifferentiating into telangiectatic osteosarcoma: importance of lytic changes and fluid cavities at imaging." by Azura et al. published in the July issue of Skeletal Radiology [1] raises a few questions that need more clarification than the information in the paper provides.

While this paper intends to demonstrate that telangiectatic osteosarcoma is the dedifferentiated component in low-grade parosteal osteosarcoma, the microscopic photographs do not adequately demonstrate that either of these components is present. There is some suggestion of these features in the imaging, but the premise of the paper and the confirmation of the imaging impression rest upon their histological correlation.

Sincerely,

Michael J. Klein, M.D.

New York, NY

\section{Reference}

1. Azura M, Vanel D, Alberghini M, Picci P, Staals E, Mercuri M. Parosteal osteosarcoma dedifferentiating into telangiectatic osteosarcoma: importance of lytic changes and fluid cavities at imaging. Skeletal Radiol. 2009;38(7):685-90. 